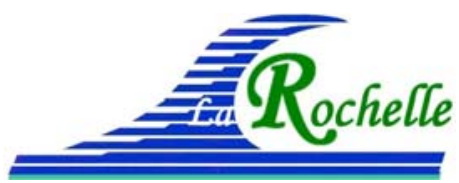

XVèmes Journées Nationales Génie Côtier - Génie Civil

La Rochelle, 29 au 31 mai 2018

DOI:10.5150/jngcgc.2018.090 @ Editions Paralia CFL

disponible en ligne - http://www.paralia.fr - available online

\title{
Cartographie du risque de submersion lié à l'élévation du niveau de la mer sur l'atoll d'Ouvéa (Nouvelle-Calédonie) : vers un outil de gestion de la zone côtière
}

\author{
Pascal DUMAS ${ }^{1}$, Sylvain LENDRE ${ }^{2}$, Matthieu LE DUF ${ }^{1}$, Michel ALLENBACH ${ }^{1}$ \\ 1. UNC, Université de la Nouvelle-Calédonie, Equipe ISEA Institut de Sciences Exactes \\ et Appliquées (EA 7484), BP R4 98851, Nouméa, Nouvelle-Calédonie. \\ pascal.dumas@univ-nc.nc
}

2. IUEM Institut Universitaire Européen de la Mer - Université de Bretagne Occidentale

\section{Résumé :}

La présente étude vise à cartographier l'emprise spatiale du risque de submersion côtière relative à l'élévation du niveau de la mer en 2100 . A cet effet, des MNT sont réalisés afin de modéliser les futures inondations qui se manifesteront dans les zones basses et plusieurs diagnostics de vulnérabilité sont établis en fonction de l'élévation du niveau marin et d'une éventuelle surcote liée aux aléas cycloniques. Ce travail permet d'éclairer les choix des décideurs et les priorités d'action concernant la politique de gestion des risques côtiers à long terme dans la perspective du changement climatique.

Mots clés : Elévation du niveau marin, submersion marine, anticipation du changement climatique, appui à la gestion intégrée des zones côtière, Nouvelle-Calédonie

\begin{abstract}
This study aims to map the projections linked to the coastal flooding risk according to sea level rise scenarios simulated by the IPCC. For this purpose, DTM are realized from topographic data so as to model the future submerged low-lying coastal zones due to sea level rise. By this approach, this work will allow enlightening the stakeholder's choices and action priorities regarding the risks management policies. In this way, this method will contribute to reduce the vulnerability of the coastal territories face to sea level rise.
\end{abstract}

Key words: Sea level rise, coastal flooding, anticipation of climate change, support for integrated coastal zone management, New Caledonia

\section{Introduction}

La plus grande partie des côtes basses et meubles du monde et particulièrement celles des petites îles de la zone intertropicale sont particulièrement exposées aux risques d'érosion côtière et de submersion marine (DUVAT et al., 2013 ; FORD, 2013). Avec l'élévation récente du niveau marin induite par le changement climatique d'origine anthropique (STOCKER et al., 2013) et l'augmentation prévue de l'intensité des phénomènes climatiques tels que les cyclones, ce sont particulièrement les atolls et des îles de faible altitude qui sont les territoires les plus vulnérables (BARNET \& 


\section{Thème 7 - Risques côtiers}

CAMPBELL, 2010 ; NUNN, 2012). Certains auteurs envisagent d'ailleurs que ce seront très vraisemblablement des espaces inhabitables à plus ou moins long terme (NURSE et al., 2014). Pour ces petits Etats insulaires, le réchauffement n'est pas une problématique abstraite. Des nations du Pacifique comme Tuvalu et Kiribati où certains atolls effleurent la surface de l'Océan à moins d'un mètre de hauteur sont déjà fortement menacés (DUVAT et al., 2013). L'érosion littorale, la submersion de zones basses, la salinisation d'aquifères côtiers sont autant de conséquences de la hausse du niveau marin auxquelles doivent faire face les îles et qui sont amenées à s'amplifier au cours du XXIe siècle.

Au regard de ce constat, une meilleure connaissance de la vulnérabilité de ces territoires littoraux aux risques liés à la mer est nécessaire. Cette dernière pourrait s'articuler au travers d'une analyse à la fois de l'évolution des milieux, des sociétés et des relations homme/milieu (DUVAT \& MAGNAN, 2010). C'est notamment dans ce cadre que depuis 2015, deux programmes de recherche INTEGRE (Etude des effets de l'érosion sur le trait de côte d'Ouvéa), financé par l'Union Européenne et MOM (Mise en place d'un réseau participatif de suivi du rivage : expérimentation dans les Iles Loyauté) du Ministère de l'Outre-mer s'intéressent aux problématiques d'érosion littorale et plus globalement à la gestion de la zone côtière des Iles Loyauté en Nouvelle-Calédonie. Cette recherche s'est particulièrement axée sur l'île basse d'Ouvéa où sa population, fortement tournée vers son lagon, s'inquiète de l'érosion du rivage. Depuis 2014, les institutions locales : Province des Iles, commune, aire coutumière, associations, en partenariat avec les acteurs coutumiers et religieux de l'île ce sont regroupées au sein d'un comité de travail afin de réfléchir ensemble aux raisons de cette érosion, de l'évolution des risques liés à la mer au regard du réchauffement climatique et des solutions qui pourraient être envisagées. Afin d'accompagner cette démarche et d'apporter des éléments scientifiques tangibles, une partie de l'île d'Ouvéa, là où les enjeux sont les plus importants, a été suivie par des campagnes régulières de mesures (profils de plages, modèles numériques de terrain MNT, photographies aériennes par drone pour restitution photogrammétrique). L'ensemble de ces levés a permis d'obtenir des données sur les impacts des phénomènes d'érosion en évolution rapide et de mieux comprendre la dynamique du littoral (LE DUFF et al., 2014, 2016a \& 2016b ; COHEN et al., 2016, ALLENBACH et al., 2016 \& 2017). Pour répondre aux attentes de la population, une partie de nos travaux s'est attachée à spatialiser les territoires vulnérables aux submersions futures compte tenu de l'élévation prévue du niveau marin induite par le réchauffement climatique. Dans le cadre de cette communication, uniquement cette partie du travail sera restituée.

\section{Présentation du site d'étude et des enjeux}

Ouvéa est l'île la plus septentrionale de l'archipel des Loyauté, située à environ $90 \mathrm{~km}$ au nord-est de la Grande-Terre de la Nouvelle-Calédonie. Sa population était de 3374 


\section{XVèmes Journées Nationales Génie Côtier - Génie Civil \\ La Rochelle, 29 au 31 mai 2018}

habitants en 2014. C'est est un atoll corallien en forme de croissant basculé vers l'ouest. Sa superficie est de $132 \mathrm{~km}^{2}$. Du fait de son inclinaison, la bordure orientale de l'île d'Ouvéa est plus élevée et longée par une série de falaises coralliennes pouvant atteindre $30 \mathrm{~m}$. L'île est bordée par un récif frangeant du côté de l'océan Pacifique et protège le rivage de la façade occidentale des houles modales de secteur sud-est. La quasi-totalité de la frange lagonaire est longée par une plage de sable fin corallien longue de $25 \mathrm{~km}$, où l'altitude est relativement basse. De par sa configuration géographique, la côte ouest de l'île est particulièrement vulnérable aux aléas météomarins (coups de vent fort de secteur ouest, cyclones et tsunamis). Le long de cette façade, les zones à risque définies comme étant situées à moins de $500 \mathrm{~m}$ du rivage et à une altitude inférieure à $10 \mathrm{~m}$ concentrent $40 \%$ de la population résidente, $50 \%$ des Installations Classées pour la Protection de l'Environnement, 50\% des établissements recevant du public et $80 \%$ des structures touristiques. Aux risques de tsunamis et cyclones s'ajoute aussi par endroit l'érosion côtière. Celle-ci est aujourd'hui particulièrement marquée à l'extrême nord de l'île à Hnyimëk (jusqu'à $-1,1 \mathrm{~m} / \mathrm{an}$ de 1954 à 2012) et à proximité du pont de Lékine dans le sud (jusqu'à -1,4 m/an de 1976 à 2012) (LE DUFF et al., 2014). Le site d'étude se situe au niveau du district de SaintJoseph occupant une zone basse au nord de l'atoll et présentant trois zones d'habitation principales réparties en tribus : Heo, Takedji et Weneki (figure 1). Les deux premières se trouvent en bord de mer et longent la côte sur environ $800 \mathrm{~m}$. Elles sont aménagées par un ensemble hétérogène de constructions légères (cases, farés) et dures (maisons, écoles, églises) s'agençant de part et d'autre d'une petite route côtière. Weneki est implantée dans une zone basse inférieure à $2 \mathrm{~m}$ d'altitude à environ $2 \mathrm{~km}$ du rivage. Le district de Saint-Joseph majoritairement sous une altitude de $5 \mathrm{~m}$ avec l'essentiel de son bâti se trouvant entre 0 et $2 \mathrm{~m}$ d'altitude est particulièrement vulnérable au risque de submersion marine.
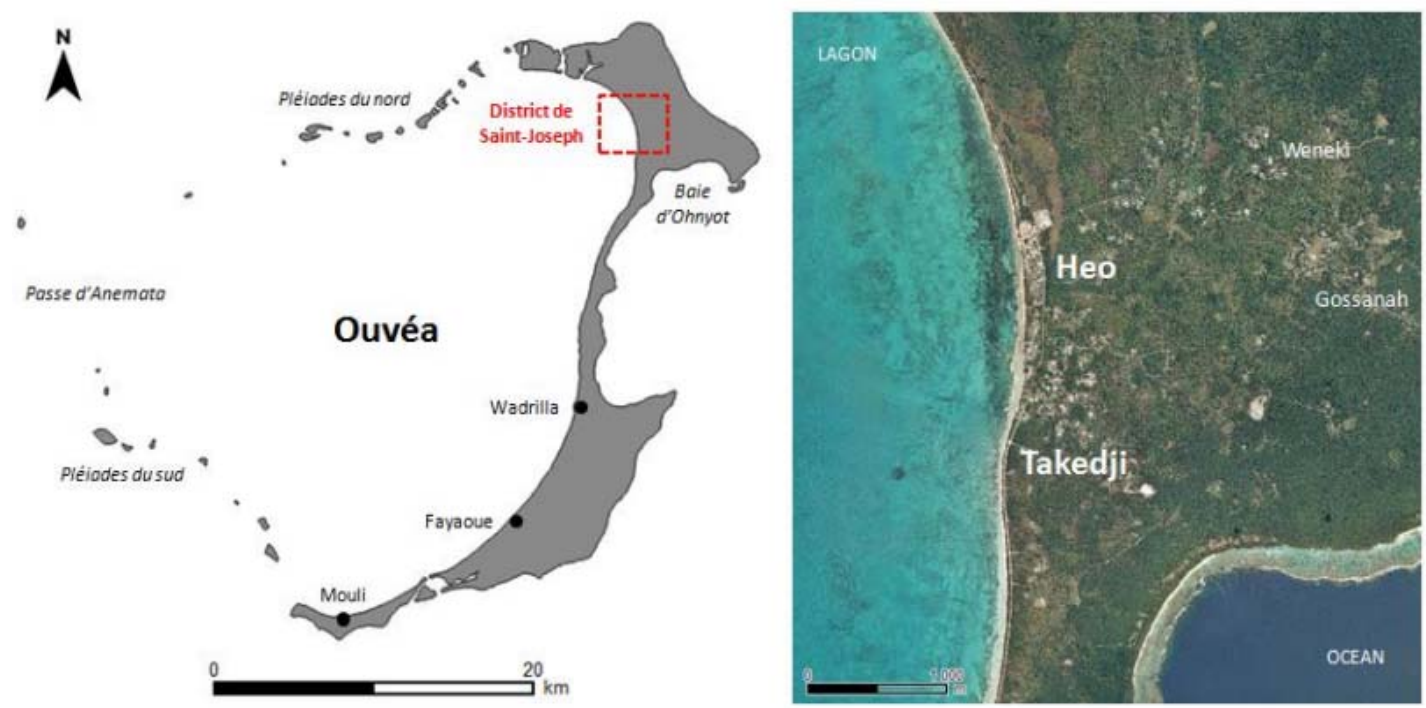

Figure 1. Localisation du site d'étude (source : DITTT; réalisation LENDRE, 2017). 


\section{Thème 7 - Risques côtiers}

\section{Matériel et méthodes}

Dès le milieu du XXe siècle de nombreux scientifiques ont cherché à comprendre le comportement du littoral et de la côte face à une élévation du niveau de la mer et de nombreux modèles ont été mis en œuvre pour le simuler (DUBOIS, 1995 ; COOPER \& PILKEY, 2004). La plupart de ces modèles reposent sur la règle dite de BRUUN (1962), empiriquement établie à partir de la variation de niveau des grands lacs américains. La loi de BRUUN est un modèle morpho-dynamique simple, basée sur une équation reliant l'élévation du niveau de la mer $\Delta \xi$ avec la pente de la plage $\beta$ afin de prédire l'évolution du trait de côte $\Delta \mathrm{S}$, en dehors des autres paramètres affectant eux aussi son évolution (LE COZANNET et al., 2014) : $\Delta \mathrm{S}=\mathrm{f} \xi(\Delta \xi)=\Delta \xi / \tan (\beta)$

Toutefois, dans un contexte géographique sur les Iles Loyauté où les données de référence ne se sont pas disponibles et du fait que les concepts sur lesquels se fonde cette règle présentent plusieurs lacunes (PILKEY et al., 1993 ; THIELER et al., 2000), nous avons préféré baser notre étude sur des méthodes plus simples à mettre en œuvre tel que le principe de l'inondation. La méthodologie développée repose ainsi sur la représentation de l'élévation du niveau marin par des modèles de submersion passive utilisés notamment dans les travaux de MIMURA (1999) ou BRUNEL et SABATIER (2007, 2009). Dans ce sens, on se limite à la projection cartographique de l'étendue d'une inondation passive sur une zone basse, potentiellement constatée sous un certain niveau d'eau atteint. Dans le cadre d'un tel modèle, on suppose que la pente du profil de la plage reste identique dans le temps et migre horizontalement vers la terre lorsque le niveau de la mer monte. Sous l'effet de l'élévation du niveau marin, la morphodynamique du système côtier de la zone d'étude à l'échelle du temps n'est pas ainsi remise en question. Hors de plus en plus d'études vont à l'encontre de ce postulat (STIVE et al., 2002 ; LOWE et al., 2010). L'interprétation de nos résultats devra donc prendre en compte ces considérations.

La mise en œuvre d'un modèle d'inondation passive présente l'avantage d'être relativement simple à mettre en œuvre bien qu'il nécessite la création d'un Modèle Numérique de Terrain (MNT). Ce dernier qui est la représentation numérique en trois dimensions $(\mathrm{x}, \mathrm{y}, \mathrm{z})$ de la topographie du sol doit être suffisamment fourni et précis pour rendre compte le plus fidèlement possible de la localisation et de la répartition des zones basses soumises au risque de submersion. Cette condition nécessite de disposer de données topographiques fines du terrain d'étude, hors les seules données disponibles au niveau du relief à Ouvéa sont celles de la BD Topo au 1/10 000 produite par la DITTT (Direction de le Topographie et des Transports Terrestres, équivalent de l'IGN en France). Cette maille de $10 \mathrm{~m}$ n'étant pas adaptée à nos travaux car trop grande, il a fallu réaliser un semi de points topographiques sur l'ensemble du district de SaintJoseph. Deux méthodes d'acquisition de données de précision centimétrique ont été utilisées. La première a consisté à réaliser un arpentage classique selon un semis de points répartis en transects de plage réguliers et perpendiculaires à la ligne de rivage à 


\section{XVèmes Journées Nationales Génie Côtier - Génie Civil \\ La Rochelle, 29 au 31 mai 2018}

l'aide d'un GPS Trimble R4 différentiel paramétré en mode RTK (Real Time Kinematic). La seconde repose sur des prises de vue aériennes de très haute résolution spatiale réalisées par un drone de type quadricopter DJI Phantom IV® équipé d'un capteur image $4 \mathrm{~K}$ fixé à une nacelle de stabilisation intégrée. Par traitements photogrammétriques avec le logiciel Agisoft Photoscan ${ }^{\circledR}$ l'information topographique et des modèles numériques surfaciques de résolution centimétrique sont restitués (COHEN et al., 2016). Les données altitudinales intégrées dans la construction du MNT réunissent ainsi les levés topographiques et photogrammétriques effectués en juin 2017, le fichier de points côtés provenant de la BD Topo ainsi que des levés additionnels DGPS effectués de façon aléatoire, à l'intérieur des terres afin d'affiner le semi de points des zones peu renseignées. Le MNT de la zone d'étude s'étendant sur 500 ha est réalisé avec le logiciel Surfer ${ }^{\circledR}$ permettant d'effectuer une interpolation par krigeage des valeurs altimétriques $\mathrm{z}$ afin de les ordonner sous la forme d'une grille dont la maille sera plus ou moins espacée en fonction de la densité du semi de points. L'ensemble de l'information géographique est restitué dans le référentiel géodésique de la NouvelleCalédonie (RGNC 1991-93 / Lambert New Caledonia).

Dans le cadre de ces travaux deux cartographies de submersion passive ont été réalisées : celle prévue par l'évolution future du niveau marin établie par les dernières projections du GIEC à l'horizon 2100 pour la région du sud-ouest Pacifique et celle relative à une surcote liée à un aléa cyclonique. En ce qui concerne la première, d'après CHURCH et al. (2013), les projections d'élévation du niveau marin sont estimées à environ $45 \pm 5 \mathrm{~cm}$ pour le premier scénario RCP2.6, à $55 \pm 5 \mathrm{~cm}$ pour les scenarii RCP4.5 et RCP6.0 et à $65 \pm 5 \mathrm{~cm}$ pour le scénario RCP8.5. Des élévations sensiblement proches ont été estimées par d'autres études (NICHOLLS \& MIMURA, 1998). Nous retiendrons uniquement ce dernier scénario certes le plus pessimiste, mais aussi l'un des plus probables car il correspond à la prolongation des émissions actuelles. Dans un second temps, à cette première submersion est aussi modélisé un potentiel effet de surcote. Ce dernier qui correspond à une élévation du niveau de la mer résultant d'une chute de la pression atmosphérique (environ $1 \mathrm{~cm}$ pour $1 \mathrm{hPa}$ ) et de violents vents d'afflux provoquant une onde de tempête (MERCIER, 2016) doit aussi être pris en compte avec les prévisions d'amplification future de l'intensité des événements météorologiques extrêmes tels que les cyclones. Combinée à de hautes marées astronomiques, les dégâts causés par la submersion lors d'un épisode cyclonique risquent d'être décuplés, d'autant plus que dans ces conditions le set-up et le run-up des vagues sont plus importants et peuvent représenter selon certains modèles jusqu'à $10 \%$ de la surcote (HUBBERT \& MCINNES, 1999). Face à ces considérations et en s'appuyant sur les études de DES GARETS (2005) et DAMLAMIAN et al. (2013) une hauteur de houle cyclonique de 1 mètre a été retenue dans l'éventualité d'un épisode de forte intensité (catégorie 4 ou 5 sur l'échelle de Saffir-Simpson). Les modèles de submersion sont établis à partir d'une marée de vives eaux équivalente au niveau atteint 


\section{Thème 7 - Risques côtiers}

par les plus hautes mers astronomiques (PHMA). Afin de connaître leur niveau, le zéro hydrographique est mesuré à partir du zéro RGNC grâce au dossier RAM (Références Altimétriques Maritimes) du SHOM. Il est situé 1,0 m en-dessous du zéro RGNC et le niveau PHMA observé dans l'atoll d'Ouvéa (au marégraphe de Hwaadrilla) atteint 1,66 m (SHOM, 2016). La valeur de 0,66 m est donc soustraite du MNT réalisé sur notre zone d'étude afin de créer un nouveau MNT possédant un niveau de base plus haut que le précédent car correspondant au niveau PHMA atteint. La spatialisation des submersions est alors simulée à partir de ce MNT.

\section{Résultats}

Dans le cadre d'une submersion passive liée à l'élévation de $+0,65 \mathrm{~m}$ à l'horizon 2100 , dans les conditions topographiques et morpho-dynamiques actuelles, se sont 60 hectares des régions basses du district de Saint-Joseph, comprenant des zones d'habitation et des terres agricoles, qui seront potentiellement inondées (figure 2). Compte tenu des caractéristiques topographiques du site mises en évidence par le MNT, Saint-Joseph subirait manifestement des intrusions d'eau marine par l'intermédiaire des zones de très faible altitude situées au nord et au sud du district. Ces zones font office d'ouverture entre le lagon et l'intérieur des terres et permettent l'engouffrement de la mer. La montée du niveau marin impliquera aussi un recul du trait de côte le long de la frange lagonaire, ce qui viendrait menacer les premières rangées d'habitation qui se situent en bord de mer. Toutefois, une grande partie de l'habitat étant à une altitude supérieure à 2 $\mathrm{m}$ sera préservé. Certaines infrastructures telles que le dispensaire et la mairie annexe de Saint-Joseph, au nord de la tribu de Heo, ainsi que les réseaux routier et électrique seront affectés. Ce sont surtout les zones basses marécageuses entourant la bande de terre surélevée allant de Takedji à Heo qui seront inondées. L'élévation du niveau marin aura donc comme conséquence l'isolement géographique de cette fine bande de terre où se concentrent l'habitat et la population. Au final, $20 \%$ des terres coutumières de la tribu de Takedji et $25 \%$ de celles de Heo devront manifestement être relocalisées d'ici la fin du XXIe siècle, en raison de l'élévation future du niveau de la mer.

La prise en compte d'une surcote de +1 mètre relative à un aléa cyclonique de forte intensité, provoquera quant à elle l'inondation de plus de 80 ha des terres. Cette submersion affectera davantage les zones vulnérables décrites ci-dessus. Les espaces habités et les plantations impactées par l'aléa cyclonique représenteraient respectivement 14 et environ 1 ha. Dans l'éventualité d'un déferlement de la houle cyclonique poussée par des vents violents à l'intérieur de la cuvette située entre Heo et Weneki, le bilan s'élèverait à 21 ha de zones d'habitation et plus de 5 ha d'exploitations agricoles menacés, pour une surface terrestre inondée d'environ 150 ha.

Enfin, le dernier scénario simule une surcote de 1 mètre liée à un aléa cyclonique lors d'une marée de vives eaux en 2100, période à laquelle le niveau marin s'élèvera à une hauteur de $65 \mathrm{~cm}$ au-dessus de l'actuel. Dans cette éventualité et dans les conditions 


\section{XVèmes Journées Nationales Génie Côtier - Génie Civil \\ La Rochelle, 29 au 31 mai 2018}

topographiques et morpho-dynamiques actuelles, l'inondation marine recouvrira plus de 300 ha de terres (figure 3 ).

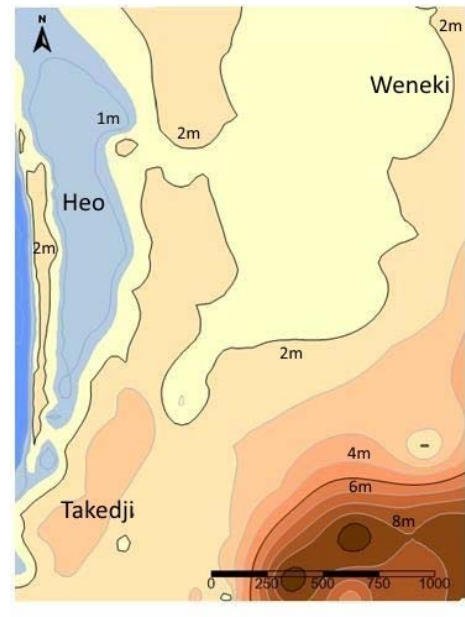

Altitude
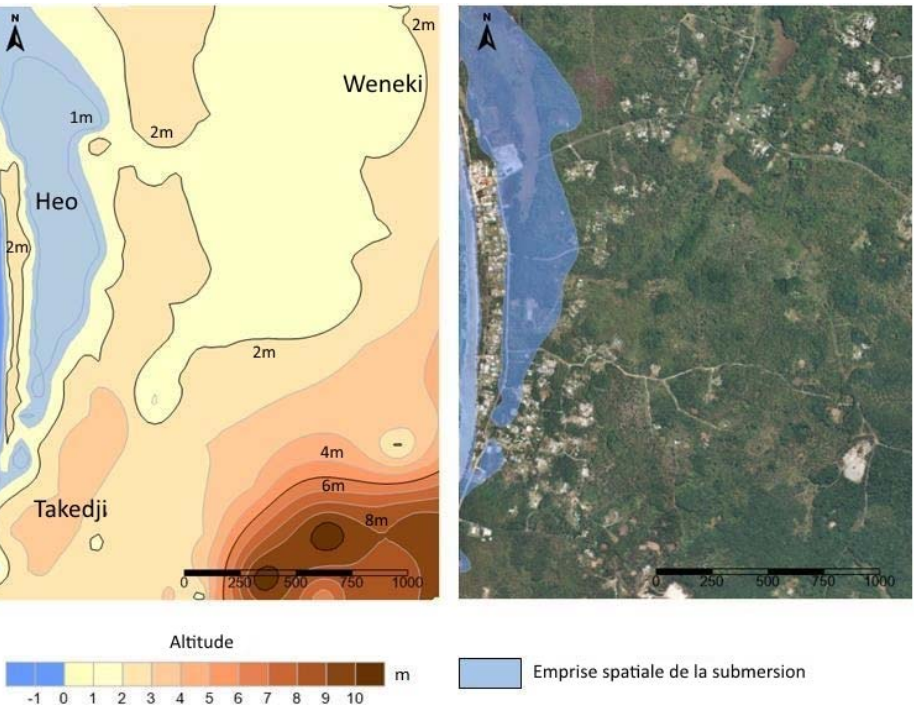

Emprise spatiale de la submersion

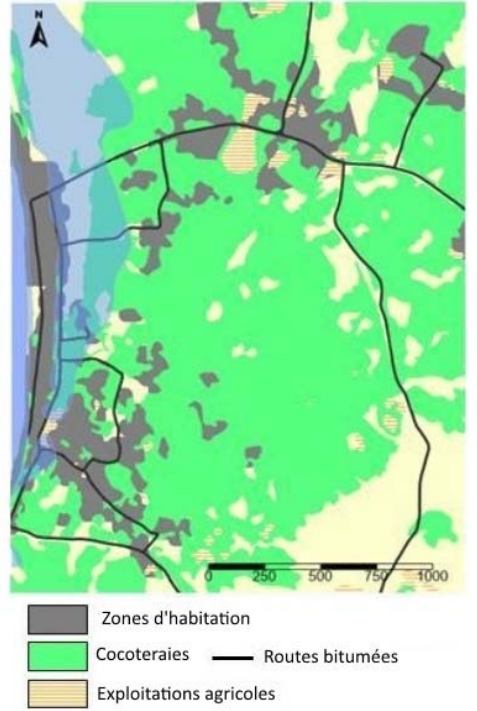

xploitations agricoles

Figure 2. Spatialisation de l'étendue probable des inondations relatives à une élévation du niveau marin de +0,65m pour l'horizon 2100 (sources : BD Topo et Ortho DITTT, données topographiques mission terrain juin 2017 ; réalisation LENDRE, 2017).

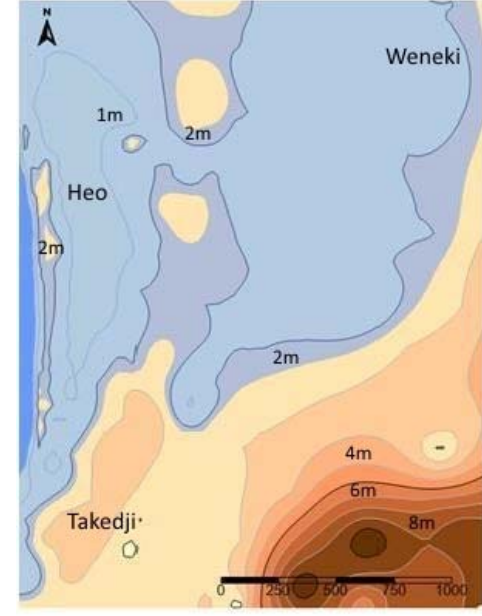

Altitude

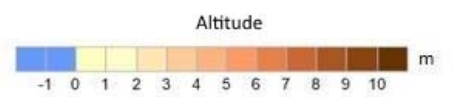

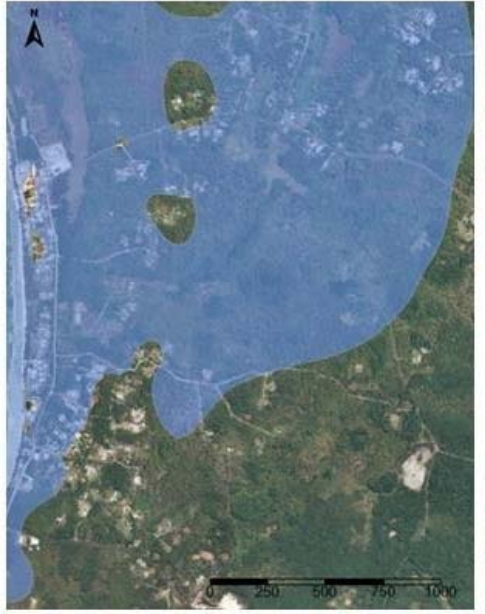

Emprise spatiale de la submersion
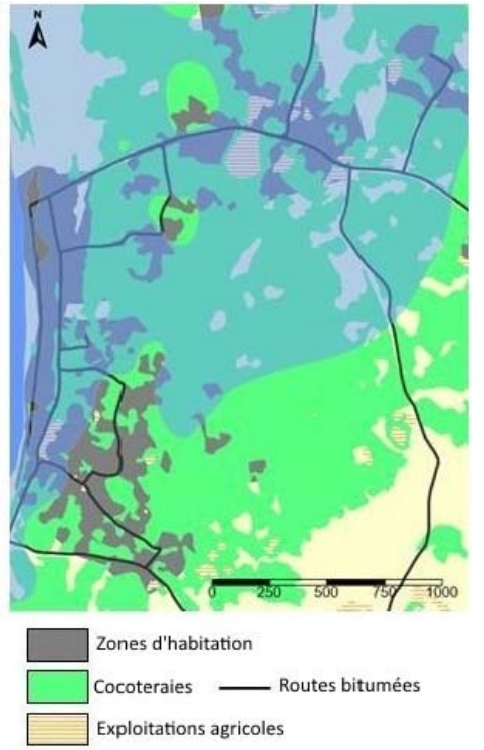

Figure 3. Spatialisation d'une submersion marine, dans le cadre d'une surcote cyclonique de $+1,0 \mathrm{~m}$ à la fin du XXIe siècle $(+0,65 \mathrm{~m} /$ niveau actuel) (sources : $B D$ Topo et Ortho DITTT, données topographiques mission terrain 2017; réalisation LENDRE, 2017). 


\section{Thème 7 - Risques côtiers}

Dans ce cas de figure, la fine bande terrestre présentant une élévation locale de 2 à $3 \mathrm{~m}$ et s'étirant des tribus de Takedji à Heo serait presque entièrement submergée par la houle cyclonique portée par l'action des vents violents et des effets de run-up associés. Avec une telle élévation de la mer, la tribu de Weneki située au nord-est du district dans une zone de cuvette serait elle aussi sous les eaux. Pour ce dernier scénario, les tribus de Heo et Weneki seraient les plus vulnérables avec 90\% de leur territoire menacé d'être inondé contre $45 \%$ pour Takedji. Au total 54 ha de zones d'habitation et 8 ha d'exploitations agricoles seraient directement impactés. Seules les maisons et cases situées sur les hauteurs de Takedji au sud-est de la tribu seraient préservées.

\section{Discussion et conclusion}

Cette étude a été proposée pour mettre en évidence les zones côtières vulnérables à une future élévation du niveau marin. La restitution cartographique repose sur des modèles de submersion passive, où l'étendue de l'inondation est représentée par l'affleurement du niveau d'eau atteint dans les conditions morpho-dynamiques actuelles du secteur d'étude. Cependant, alors que la submersion par un effet de surcote cyclonique peut correspondre aux projections spatiales d'une augmentation instantanée et éphémère du niveau marin en fonction de la topographie du site, la modélisation d'une élévation du niveau marin associée au changement climatique intègre quant à elle des processus multiples et plus lents, susceptibles de provoquer de nombreuses reconfigurations morphologiques du profil de plage et de la côte. Dans cette dernière perspective, ces potentielles modifications relatives aux dynamiques sédimentaires et au recul du trait de côte engendré par la hausse du niveau marin n'ont pas été prises en compte et doivent être considérées dans l'interprétation des résultats. La réponse du littoral aux variations du niveau de la mer n'est clairement pas linéaire et ce dernier se façonne plus encore lors d'évènements à haute énergie tels que des dépressions ou cyclones (ou du moins les périodes de mer agitée avec des houles, ou des vagues, brisant sur la plage) qui agissent comme des processus essentiels.

Au-delà de ces considérations, l'intérêt de ce travail réside davantage dans la spatialisation des zones basses les plus prédisposées à l'ennoiement en établissant un premier diagnostic de vulnérabilité face au risque de submersion. Ainsi, dans le cadre d'une gestion à moyen/long terme des enjeux humains et matériels du district de SaintJoseph, ces simulations permettent de sensibiliser les populations locales aux futurs risques côtiers qui affecteront leur territoire, en évaluant et spatialisant les dommages futurs. Par cette approche, cette cartographie s'inscrit dans une perspective de réalisation d'outils d'aide à la décision, destinés aux décideurs, gestionnaires et aux populations en termes d'aménagement et de gestion des territoires côtiers. Dans le cadre spécifique des Iles Loyauté, constituées de terres coutumières réputées incessibles, incommutables, insaisissables et inaliénables, la gestion du foncier relève avant tout des autorités coutumières, premier acteur de la gestion du littoral. Le développement de ce 


\section{XVèmes Journées Nationales Génie Côtier - Génie Civil \\ La Rochelle, 29 au 31 mai 2018}

type d'outil est donc également le meilleur moyen d'initier une concertation efficace avec ces dernières afin de les accompagner vers une organisation adaptée et une occupation plus raisonnée de leur territoire mais aussi d'envisager d'éventuels déplacements de populations vers des zones sécurisées, dans la perspective du changement climatique.

\section{Références bibliographiques}

ALLENBACH M., LE DUFF M., DUMAS P., COHEN O. (2016). Gestion intégrée, risques côtiers et aménagements sur les littoraux océaniens français. XIV ${ }^{\mathrm{èmes}}$ Journées Nationales Génie Côtier-Génie Civil, pp 127-136. https://doi.org/10.5150/jngcgc.2016.015

ALLENBACH M., LE DUFF, M., DUMAS, P., BOUTEILLER Y. (2017). Advantages and challenges of participatory management of customary coastal areas in the French Islands of the Pacific, In Leal Filho, W. (Ed) Climate Change Impacts and Adaptation Strategies in Coastal Communities. Ed. Springer, pp 299-313.

BARNETT J., CAMPBELL J. (2010). Climate change and small island States: power, knowledge in the South Pacific, Earthscan, Londres, UK, 218 p.

BRUNEL C., SABATIER F. (2007). Pocket beach vulnerability to sea-level rise, Journal of Coastal Research, $n^{\circ} 50$, pp 604-609.

BRUNEL C., SABATIER F. (2009). Potential influence of sea-level rise in controlling shoreline position on Mediterranean Coast, Geomorphology, Vol. 107(1-2), pp 47-57. https://doi.org/10.1016/j.geomorph.2007.05.024

BRUUN P. (1962). Sea level rise as a cause of shore erosion. Journal of the Waterways and Harbours Division, Vol. 88(1), pp117-132.

CHURCH J.A., CLARK P.U., CAZENAVE A., GREGORY A., JEVREJEVA S., LEVERMANN A., MERRIFIELD M.A., MILNE G.A., NEREM R.S., NUNN P.D., PAYNE A.J., PFEFFER W.T., STAMMER D. UNNIKRISHNAN A.S. (2013). Sea Level Change. In: Climate Change 2013, The Physical Science Basis. Contribution of Working Group to the 5th Assessment Report of the IPCC, Cambridge Univ Press.

COHEN O., DUMAS P., LE DUFF M., ALLENBACH M. (2016). Etude du rivage par photogrammétrie aéroportée à très haute résolution spatiale. Exemple à Ouvéa, Nouvelle Calédonie. XIV ${ }^{\text {èmes }}$ Journées Nat. Génie Côtier-Génie Civil, pp 361-370. https://doi.org/10.5150/jngcgc.2016.041

COOPER J.A.G., PILKEY O.H. (2004). Longshore drift, trapped in an expected universe. J. of Sed. Res., Vol. 74, pp 599-606.

DAMLAMIAN H., KRUGER J., TURAGABECI M., KUMAR S. (2013). Modélisation des submersions marines d'origine cyclonique dans les atolls d'Apataki, Arutua, Kauehi, Manihi et Rangiroa, en Polynésie française, Rapport technique, CPS, Division géosciences et technologies appliquées, 66 p.

DES GARETS E. (2005). Bilan des connaissances sur les surcotes marines en Polynésie, Rapport BRGM, référence RP-55038-FR 


\section{Thème 7 - Risques côtiers}

DUBOIS R.N. (1995). The transgressive barrer model: an alternative to twodimensional volume balanced models, Journ. Coast. Res., Vol. 11, pp 272-1286.

DUVAT V., MAGNAN A. (2010). Des archipels en péril ? Les Maldives et les Kiribati face au changement climatique, VertigO, Vol. 10, 3, http://vertigo.revues.org.

DUVAT V., MAGNAN A., POUGET F. (2013). Exposure of atoll population to coastal erosion and flooding: a South Tarawa assessment, Kiribati, Sustainable Sciences, Understanding and managing global change in small islands, ${ }^{\circ} 8$, pp 423-440. FORD M. (2013). Shoreline changes interpreted from multi-temporal aerial photographs and high resolution satellite images: Wotje Atoll, Marshall Islands, Remote Sensing of Environment, $\mathrm{n}^{\circ} 135$, pp130-140.

HUBBERT G.D., MCINNES K.L. (1999). A storm surge inundation model for coastal planning and impact studies, Journal of Coastal Research, ${ }^{\circ} 15$, pp 168-185.

LE COZANNET G., GARCIN M., YATES M., IDIER D., MEYSSIGNAC B. (2014). Approaches to evaluate the recent impacts of sea-level rise on shoreline changes, EarthScience Reviews, $\mathrm{n}^{\circ}$ 138, pp 47-60. https://doi.org/10.1016/i.earscirev.2014.08.005

LE DUFF M., DUMAS P., COHEN O., ALLENBACH M. (2016a). Coastal erosion monitoring on Ouvea island (New Caledonia): involving the local community in climate change adaptation, In Leal Filho, W. (Ed) Climate Change Adaptation in Pacific Countries, Ed. Springer, pp 255-268

LE DUFF M., DUMAS P., SABINOT C., ALLENBACH M. (2016b). Le risque tsunami en NC: Évolutions des facteurs de vulnérabilités et de résiliences à Lifou en territoire kanak, VertigO Vol.16-3

LE DUFF M., DUMAS P., ALLENBACH M. (2014). Quelle gouvernance des risques littoraux aux îles Loyauté (NC), dans le cadre du réchauffement climatique ? In Proceedings International Conference Coastal Risks: Hazards, Issues, Representations, Management, pp 429-436.

LOWE J.A., WOODWORTH P.L., KNUTSON T., MCDONALD R.E., MCINNES K. L., WOTH K., VON STORCH H., WOLF J., SWAIL V., BERNIER N. B., GULEV S., HORSBURGH K.J., UNNIKRISHNAN A.S., HUNTER J.R., WEISSE R. (2010). Past and future changes in extreme sea levels and waves, In: Understanding Sea-Level Rise and Variability [Church, J. A., Woodworth, P. L., Aarup, T., Wilson, W. S. (eds.)], Wiley-Blackwell, Hoboken, NJ, USA, pp 326-375. https://doi.org/10.1002/9781444323276.ch11

MERCIER Y. (2016). Phénomène vague-submersion marine, Comité utilisateur OBLIC, DIMENC, Division Prévision Marine de Météo France, Météo NC, 53 p.

MIMURA (1999). Vulnerability of island countries in the South Pacific to sea level rise and climate change, Climate Research, Vol. 12, pp 137-143. https://doi.org/10.3354/cr012137 NICHOLLS R.J., MIMURA N. (1998). Regional issues raised by sea-level rise and their policy implications, Climate Research, $\mathrm{n}^{\circ} 11$, pp 5-18.

NUNN P.D. (2012). Climate change and Pacific Island Countries, Asia-Pacific Human Development Report Background Papers Series, UNDP, 97 p. 


\section{XVèmes Journées Nationales Génie Côtier - Génie Civil \\ La Rochelle, 29 au 31 mai 2018}

NURSE L.A., McLEAN R.F., AGARD J., BRIGUGLIO L.P., DUVAT-MAGNAN V., PELESIKOTI N., TOMPKINS E., WEBB A. (2014). Chapter 29: Small islands. In: Climate Change 2014. Impacts, Adaptation, and Vulnerability. Contribution of Working Group II to the 5th Assessment Report of IPCC, Cambridge Univ Press, pp 1613-1654.

PILKEY O.H., YOUNG R.S., RIGGS S.R., SMITH A.W.S., WU H., PILKEY W.D. (1993). The concept of shoreface profile of equilibrium: a critical review. J. Coastal Research 9 (I), pp 255-278.

SHOM (2016). Edition 2016 du produit Références Altimétriques Maritimes (RAM).

STIVE M.J.F., AARNINKHOF S.G.J., HAMM L., HANSON H., LARSON M., WIJNBERG K.M., NICHOLLS R.J., CAPOBIANCO M. (2002). Variability of shore and shoreline evolution. Coastal Engineering, Vol. 47, $\mathrm{n}^{\circ} 2, \mathrm{pp}$ 211-235. https://doi.org/10.1016/S0378-3839(02)00126-6

STOCKER T.F., QIN D., PLATTNER G.-K., TIGNOR M., ALLEN S.K., BOSCHUNG J., NAUELS A., XIA Y., BEX V., MIDGLEY P.M. (2013). Climate Change 2013: Synthesis Report. Contribution of Working Group I to the 5th Assessment Report of the IPCC, Cambridge Univ Press, 204 p.

THIELER E.R., PILKEY O.H., YOUNG R.S., BUSH D.M., CHAI F. (2000). The use of mathematical models to predict beach behavior for US Coastal Engineering: a critical review. J. of Coastal Res., Vol. 16, pp 48-70. 
Thème 7 - Risques côtiers 